\title{
Evidence For COVID-19 Vaccine Deferred Dose 2 Boost Timing
}

\author{
David E. Epperly ${ }^{1}$ \\ Created, Copyright 2021.0101a \\ Revised, Copyright 2021.0105c
}

\section{Abstract}

Both Pfizer and Moderna vaccine trials used accelerated methods to achieve rapid FDA approval with minimal and wise compromises. While many established vaccines use boost / dose 2 timings that are typically in the 3 to 120 months range ${ }^{2}$, dose 2 timings for both trials were less than 30 days as part of a crucial effort to rapidly develop a vaccine with significant efficacy. Data from both trials showed excellent dose 1 efficacy; however, neither of the related Safety and Efficacy publications highlighted this dose 1 efficacy excellence.

The trials, manufacturing, and distribution programs have rapidly delivered millions of doses to points of care. However, the ability to administer these doses at localities as rapidly as they can be distributed has been shown as the largest current challenge to achieving the widespread vaccination. Improvements could reduce mortality rates that presently exceed 2000 per day, avoid serious cases at a time where hospital census is at full capacity or beyond, and minimize long-term sequelae.

This study suggests that it is possible to achieve these and other goals by recognizing the evidence for COVID-19 vaccine deferred boost timing and using a more conventional, decades-tested, boost timing on the order of months. This would enable currently limited US resources to effectively double the population being vaccinated over the next few months, enabling more rapid vaccination of those at highest-risk for severe infections.

\section{Methods}

This meta study selected vaccines approved under US FDA EUA and evaluated the available vaccine immune response data, infection immune response data, general immunological supporting material, current vaccination plan performance, current COVID-19 infection and mortality data, existing US CDC guidance, and other bioinformatic data to understand and suggest opportunities for improved epidemiologic action.

\section{Results And Discussion}

In the Pfizer study ${ }^{3}$, there was a minor omission in the study data that presented the dose 1 data in a manner that made it difficult to correctly calculate dose 1 efficacy. Specifically, the table in Figure 3 describing "After dose 1 to before dose 2" cannot be used to properly calculate dose 1 efficacy because it does not allow any time for seroconversion, whereas the data for " $>=7$ days after dose 2 " properly allows for seroconversion. The "dose $1+7$ days to dose 2 " data was omitted. As an aside, this also creates an imbalance in the data presentation such that dose 1 and dose 2 efficacy data in the study is not properly comparable due to this difference in methods. 
In the Moderna study ${ }^{4}$, Figure 3B presents data that enables dose 1 and dose 2 efficacy data to be properly calculated using a 14 day seroconversion screen, but the dose 1 efficacy data is not presented in the article.

Given the data from the Pfizer Figure 3 chart and table, and the Moderna Figure 3B table, it is possible to reasonably calculate ${ }^{5}$ the efficacy data of dose 1 in both studies.

In the case of the Pfizer study, some data had to be extracted visually from the Figure 3 chart to arrive at the necessary data. While there is potential for minor error in visually interpreting this data from the chart, that visual chart interpretation error is likely to affect the Pfizer dose 1 efficacy calculation by less than 2 percentage points. The needed data to improve the calculations below surely exist elsewhere in Pfizer documents.

In the Moderna study, there is a small $\mathrm{N}$ of 2 vaccinated arm infections during the dose 1 period. To help understand how that small $\mathrm{N}$ affects efficacy calculation, a hypothetical addition of more vaccinated arm infections can be calculated to see the efficacy in that hypothetical case which helps quantify any concerns regarding the small $\mathrm{N}$. In a table that follows, a calculation was included for vaccinated arm infections being what was found in the study ( 2 infections) and a hypothetical case of double that number (4 infections over a 14 day period), keeping in mind that the total $\mathrm{N}$ for dose 2 was only 19 over the 120 day period.

By using the data captured but not well presented in these studies, it is possible to provide information regarding dose 1 efficacy of both vaccines as shown in Table 1:

\begin{tabular}{|c|c|c|c|c|c|c|c|c|c|c|}
\hline & $\begin{array}{l}\text { Pfizer } \\
\text { Dose } \\
\text { 2+7days } \\
\text { To End } \\
\text { (Study } \\
\text { Figure 3) }\end{array}$ & $\begin{array}{l} \\
\text { Pfizer } \\
\text { Dose } 1 \text { to } \\
\text { Dose } 2\end{array}$ & $\begin{array}{l}\text { Pfizer } \\
\text { Dose 1 + } \\
12 \text { Days } \\
\text { (approx } \\
\text { from } \\
\text { chart) to } \\
\text { Dose } 2\end{array}$ & $\begin{array}{l}\text { Pfizer } \\
\text { Dose 1 + } \\
12 \text { Days } \\
\text { (approx } \\
\text { from } \\
\text { chart) to } \\
\text { Dose 2 + } \\
7 \text { Days }\end{array}$ & $\begin{array}{l}\text { Pfizer } \\
\text { Dose 1+ } \\
12 \text { Days } \\
\text { (approx } \\
\text { from } \\
\text { chart) to } \\
\text { End }\end{array}$ & $\begin{array}{l}\text { MRNA } \\
\text { Dose } 2 \\
\text { +14 Days } \\
\text { To End } \\
\text { (Study } \\
\text { Figure } \\
\text { 3B) }\end{array}$ & $\begin{array}{l}\text { MRNA } \\
\text { Dose } 1 \\
\text { +14 Days } \\
\text { To Dose2 }\end{array}$ & $\begin{array}{l}\text { MRNA } \\
\text { Dose } 1 \\
\text { +14 Days } \\
\text { To Dose2 } \\
\text { + 14 Days }\end{array}$ & $\begin{array}{l}\text { MRNA } \\
\text { Dose } 1 \\
\text { +14 Days } \\
\text { To End }\end{array}$ & $\begin{array}{l}\text { MRNA } \\
\text { Dose 1+14 } \\
\text { Days To } \\
\text { Dose2 And } \\
\text { Simulating } \\
2 \text { More } \\
\text { IllVaccine }\end{array}$ \\
\hline TotalPlacebo & 21686 & 21686 & 21686 & 21686 & 21686 & 14598 & 14598 & 14598 & 14598 & 14598 \\
\hline TotalVaccine & 21669 & 21669 & 21669 & 21669 & 21669 & 14550 & 14550 & 14550 & 14550 & 14550 \\
\hline IIIPlacebo & 172 & 82 & 39 & 60 & 275 & 204 & 35 & 54 & 258 & 35 \\
\hline IIIVaccine & 9 & 39 & 7 & 9 & 18 & 12 & 2 & 2 & 14 & 4 \\
\hline RiskPlacebo & 0.007931 & 0.003781 & 0.001798 & 0.002767 & 0.012681 & 0.013975 & 0.002398 & 0.003699 & 0.017674 & 0.00239759 \\
\hline RiskVaccine & 0.000415 & 0.0018 & 0.000323 & 0.000415 & 0.000831 & 0.000825 & 0.000137 & 0.000137 & 0.000962 & 0.00027491 \\
\hline VE & $94.8 \%$ & $52.4 \%$ & $82.0 \%$ & $85.0 \%$ & $93.4 \%$ & $94.1 \%$ & $94.3 \%$ & $96.3 \%$ & $94.6 \%$ & $88.5 \%$ \\
\hline
\end{tabular}

While 95\% confidence intervals were not calculated here, they could be calculated using compete study datasets. Table 1 shows that the Pfizer dose 1 efficacy is likely to be near $82 \%$ and perhaps as high as $93 \%$. The Moderna vaccine dose 1 efficacy is likely to be between $88 \%$ and $94 \%$, keeping in mind the small $\mathrm{N}$ of 2 infections in the vaccine arm show a narrow time period where the efficacy calculates to $96 \%$ based on the data from "Dose 1 + 14 Days To Dose 2 + 14 Days". To accommodate the small N, the hypothetical of doubling that $\mathrm{N}$ to 4 helps determine a reasonable lower bound for dose 1 efficacy of $88 \%$. Confidence interval data could be calculated to further resolve $95 \%$ confidence limits. 
Even if dose 1 had only a 60\% efficacy, which is clearly lower than a reasonable calculation would determine, it could be argued that doubling the vaccinated population with these dose 1 efficacies would be more holistically efficacious than ensuring a $95 \%$ efficacy during a period of vaccinating those at high-risk. This is further bolstered by the vaccine arm of Moderna having found zero serious cases even during the dose 1 only period. The vaccine arm of Pfizer "at least 7 days after Dose 2" had 1 case where an individual was found to have "oxygen saturation ... 93\% on room air ... not hospitalized, did not seek further medical care". 6

Having shown good dose 1 efficacy data, the question of durability arises. From numerous COVID-19 infection immune response studies, it can be shown that antibody titers remain protective for $>3$ months $s^{7,8,9}$. Recent studies have shown protection at 6 to 8 months and suggested greater periods of durability ${ }^{10,11,12,13,14}$. Antibody titer data from both vaccines' Phase 1,2 , and 3 trial data show strong immune response ${ }^{15,16}$. Furthermore, $\mathrm{B}$ memory and $\mathrm{B} / \mathrm{T}$ related study results enable further indirect data that supports the case for at least several months of immune response durability based on similarities between infection immune response and vaccine immune response ${ }^{13}$. While it may be tempting to assume nothing and ignore correlative observations in available data, it may be wise to recall that a discomfiting lesson was learned when the WHO stated that "There is currently no evidence that people who have recovered from COVID-19 and have antibodies are protected from a second infection". All available data must be utilized to arrive at a multiple data set integrated medicine choice that is holistically efficacious when the potential to save thousands of lives per day over a period several months is a likely benefit. General immunological principles and intelligent fusion of ALL available data sources can help guide our optimizations for the present pandemic with the understanding that we should set "expectations based on prior findings while avoiding the overused approach of assuming nothing". ${ }^{17}$ While durability is improved with a 2 or more dose regimen ${ }^{18}$, dose timing is subject to optimization.

It can also be shown from general immunological principles that a level of sterilizing immunity generally arises when IgA, CD8+ T cytotoxic, and IgG are present ${ }^{19,20,21,22,14}$. Given that IgG and IgA antibody titers correlate to $\mathrm{CD} 8+\mathrm{T}$ cytotoxic cell and B / plasma cell populations (but not necessarily the inverse ${ }^{23,14}$ ), all of these taken together can be expected, from studies referenced earlier, to afford some level of sterilizing immunity which is beneficial to those not yet vaccinated. Having these in a vaccinated population of double the size could further create a significant benefit. Furthermore, using a dose 2 timing on the order of 3 to 5 months could create a nearly double wide window of high immune activity that results in some level of sterilizing immunity for a period on the order of 8 months or more; whereas the closely spaced regimen used in the study would likely be several months shorter. Optimal dose 2 timing could cause the IgG titer and other immune response dose 1 / dose 2 curves to overlap, resulting in a most favorable strength and length immune response that could support a level of longer sterilizing immunity. Based on antibody titer data referenced above, that optimal timing is likely to be far longer than 21 or 28 days.

CDC guidance has apparently considered this deferred dose 2 scenario. It states "Second doses administered within a grace period of $\leq 4$ days from the recommended date for the second dose are considered valid; however, doses administered earlier do not need to be repeated. The second dose should be administered as close to the recommended interval as possible. However, there is no maximum interval between the first and second dose for either vaccine." 24 The WHO SAGE and UK MHRA also indicated flexibility: "made a provision for countries in exceptional circumstances of vaccine supply constraints and epidemiological settings to delay the administration of the second dose for a few weeks in order to maximize the number of individuals benefiting from a first dose." ${ }^{25,26}$ and "In terms of protecting priority groups, a model where we can vaccinate twice the number of people in the next two to three months is obviously much more preferable." ${ }^{27}$ 
Current US mortality rates as of mid-December 2021 exceed an average of 2000 per day. This would equate to over 180,000 deaths over a 3 month period. Doubling the vaccinated population in that same 3 month period could likely result in dramatically lower deaths, possibly exceeding 1000 deaths per day of deferral depending upon effectiveness of vaccination priority planning.

Given:

1. Good efficacy of dose 1 for both vaccines

2. Strong likelihood of much greater than 3 month durability of dose 1

3. Ability to double the vaccinated population during the dose 2 deferral period

4. Strong likelihood of dramatic reduction in hospitalization rates

5. Likely reduction of mortality rates on the order of 1000 deaths per day of deferral

6. Potential to increase the period over which some level of sterilizing immunity could persist

7. A level of deferred dose 2 support from UK MHRA, US CDC, and WHO current guidance

8. Present hospital census overload

9. Potential for more-timely improved economic and mental health restorative cadence

10. Many decades of experience with vaccine boost schedules on the order of 3 to 120 months

11. Ability to initiate a study to monitor $\mathrm{B} / \mathrm{T}$ cell and antibody prevalence during dose 1 period

it would seem irresponsible to use the "warp speed" trial's $<30$ day dose 2 boost timing that was sharply designed to rapidly determine some understanding of vaccine efficacy, not as an optimal boost timing period.

The extensive monitoring programs that follow the vaccinated population for both of these vaccines would detect any incidence of serious infection and any observation related to dose 1 efficacy could be used by the $\mathrm{CDC}$ to trigger guidance modification that would appropriately re-prioritize timing of the $2^{\text {nd }}$ dose.

This evidence suggests the use of a 3 to 5 month dose 2 boost schedule to minimize hospitalizations and deaths over the calendar year 2021. It also suggests the utility of an appropriate study that would monitor antibody titer and B/T cell prevalence in an appropriately sized group of dose 1 recipients to further guide the timing of the $2^{\text {nd }}$ dose going forward. It also suggests urgency in adopting the improved vaccination plans suggested herein that could rapidly and significantly reduce hospitalizations and mortality.

\section{References}

\footnotetext{
${ }^{1}$ David E. Epperly, AA, President, Inflection Technologies Corporation, Torrance, CA, (Corresponding Author: editor1 [a t] reallycorrect.com)

${ }^{2}$ https://www.cdc.gov/vaccines/schedules/hcp/imz/adult.html

${ }^{3}$ Polack FP, Thomas SJ, Kitchin N, et al. Safety and efficacy of the BNT162b2 mRNA covid-19 vaccine. N Engl J Med 2020. doi:10.1056/NEJMoa2034577. https://www.neim.org/doi/full/10.1056/NEJMoa2034577?query=RP.

${ }^{4}$ Lindsey R. Baden, M.D., Hana M. El Sahly, M.D., Brandon Essink, M.D., et-al. Efficacy and Safety of the mRNA-1273 SARSCoV-2 Vaccine. N Engl J Med 2020. DOI: 10.1056/NEJMoa203538. https://www.nejm.org/doi/full/10.1056/NEJMoa2035389

${ }^{5}$ https://www.cdc.gov/csels/dsepd/ss1978/lesson3/section6.html
} 
${ }^{6}$ https://www.fda.gov/media/144245/download (pg 30) from https://www.fda.gov/advisory-committees/advisorycommittee-calendar/vaccines-and-related-biological-products-advisory-committee-december-10-2020-meetingannouncement\#event-materials

7 A. S. Iyer, F. K. Jones, A. Nodoushani, M. Kelly, M. Becker, D. Slater, R. Mills, E. Teng, M. Kamruzzaman, W. F. GarciaBeltran, M. Astudillo, D. Yang, T. E. Miller, E. Oliver, S. Fischinger, C. Atyeo, A. J. lafrate, S. B. Calderwood, S. A. Lauer, J. Yu, Z. Li, J. Feldman, B. M. Hauser, T. M. Caradonna, J. A. Branda, S. E. Turbett, R. C. LaRocque, G. Mellon, D. H. Barouch, A. G. Schmidt, A. S. Azman, G. Alter, E. T. Ryan, J. B. Harris, R. C. Charles, Persistence and decay of human antibody responses to the receptor binding domain of SARS-CoV-2 spike protein in COVID-19 patients. Sci. Immunol. 5, eabe0367 (2020). doi:10.1126/sciimmunol.abe0367pmid:33033172 https://immunology.sciencemag.org/content/5/52/eabe0367

${ }^{8}$ Isho, B., K. T. Abe, M. Zuo, A. J. Jamal, B. Rathod, J. H. Wang, Z. Li, G. Chao, O. L. Rojas, Y. M. Bang, et al. . 2020. Persistence of serum and saliva antibody responses to SARS-CoV-2 spike antigens in COVID-19 patients. Sci. Immunol. 5: eabe5511. https://immunology.sciencemag.org/content/5/52/eabe5511

${ }^{9}$ Persistence of serum and saliva antibody responses to SARS-CoV-2 spike antigens in COVID-19 patients. Baweleta Isho, Kento T. Abe, Michelle Zuo, et-al. Science Immunology08 Oct 2020. DOI: 10.1126/sciimmunol.abe5511. https://immunology.sciencemag.org/content/5/52/eabe5511

${ }^{10}$ Orthogonal SARS-CoV-2 Serological Assays Enable Surveillance of Low-Prevalence Communities and Reveal Durable Humoral Immunity. Ripperger, Tyler J. et al. Immunity, Volume 53, Issue 5, 925 - 933.e4.

https://www.cell.com/immunity/fulltext/S1074-7613(20)30445-3

${ }^{11}$ Robust SARS-CoV-2-specific T-cell immunity is maintained at 6 months following primary infection. J Zuo, A Dowell, H Pearce, K Verma, HM Long, J Begum, F Aiano, Z Amin-Chowdhury, B Hallis, L Stapley, R Borrow, E Linley, S Ahmad, B Parker, A Horsley, G Amirthalingam, K Brown, ME Ramsay, S Ladhani, P Moss bioRxiv 2020.11.01.362319; doi: https://doi.org/10.1101/2020.11.01.362319

12 Immunological memory to SARS-CoV-2 assessed for greater than six months after infection. Jennifer M. Dan, Jose Mateus, View ORCID ProfileYu Kato, Kathryn M. Hastie, Caterina E. Faliti, Sydney I. Ramirez, April Frazier, Esther Dawen Yu, Alba Grifoni, Stephen A. Rawlings, Bjoern Peters, Florian Krammer, Viviana Simon, Erica Ollmann Saphire, Davey M. Smith, Daniela Weiskopf, Alessandro Sette, View ORCID ProfileShane Crotty doi: https://doi.org/10.1101/2020.11.15.383323

${ }^{13}$ Rapid generation of durable B cell memory to SARS-CoV-2 spike and nucleocapsid proteins in COVID-19 and convalescence. By Gemma E. Hartley, Emily S.J. Edwards, Pei M. Aui, Nirupama Varese, Stephanie Stojanovic, James McMahon, Anton Y. Peleg, Irene Boo, Heidi E. Drummer, P. Mark Hogarth, Robyn E. O'Hehir, Menno C. van Zelm. Science Immunology22 Dec 2020 Memory B cells specific for SARS-CoV-2 spike and nucleocapsid proteins persist in peripheral blood after recovery from COVID-19 https://immunology.sciencemag.org/content/5/54/eabf8891

${ }^{14}$ https://reallycorrect.com/ReallyCorrectWp1/immunology-vaccines-viral-loads-symptom-severity-and-immunity/\#COVID19-Immunity

${ }^{15}$ Corbett KS Flynn B Foulds KE et al.Evaluation of the mRNA-1273 vaccine against SARS-CoV-2 in nonhuman primates. N Engl J Med. 2020; (published online July 28.) https://doi.org/10.1056/NEJMoa2024671

${ }^{16}$ Sahin, U., Muik, A., Derhovanessian, E. et al. COVID-19 vaccine BNT162b1 elicits human antibody and TH1 T cell responses. Nature 586, 594-599 (2020). https://doi.org/10.1038/s41586-020-2814-7

${ }^{17}$ Nicole Baumgarth, Janko Nikolich-Žugich, F. Eun-Hyung Lee and Deepta Bhattacharya, J Immunol September 4, 2020, ji2000839; DOI: https://doi.org/10.4049/jimmunol.2000839

${ }^{18}$ https://reallycorrect.com/ReallyCorrectWp1/immunology-vaccines-viral-loads-symptom-severity-and-immunity/

${ }^{19}$ Dutta, A; Huang, CT; Lin, CY; Chen, TC; Lin, YC; Chang, CS; He, YC (6 September 2016). "Sterilizing immunity to influenza virus infection requires local antigen-specific T cell response in the lungs". Scientific Reports. 6: 32973. 
Bibcode:2016NatSR...632973D. doi:10.1038/srep32973. PMC 5011745. PMID 27596047.

https://www.nature.com/articles/srep32973

${ }^{20}$ Enhanced SARS-CoV-2 neutralization by dimeric IgA. By Zijun Wang, Julio C. C. Lorenzi, Frauke Muecksch, Shlomo Finkin, Charlotte Viant, Christian Gaebler, Melissa Cipolla, Hans-Heinrich Hoffmann, Thiago Y. Oliveira, Deena A. Oren, Victor Ramos, Lilian Nogueira, Eleftherios Michailidis, Davide F. Robbiani, Anna Gazumyan, Charles M. Rice, Theodora Hatziioannou, Paul D. Bieniasz, Marina Caskey, Michel C. Nussenzweig Published Online07 Dec 2020 DOI:

10.1126/scitranslmed.abf1555. https://stm.sciencemag.org/content/early/2020/12/04/scitranslmed.abf1555

${ }^{21}$ https://pipelinereview.com/index.php/2020090875801/Vaccines/Vaxarts-Oral-COVID-19-Vaccine-Candidate-InducesPotent-Systemic-and-Mucosal-Immune-Responses-in-Preclinical-Studies.html

${ }^{22}$ https://reallycorrect.com/ReallyCorrectWp1/immunology-vaccines-viral-loads-symptom-severity-andimmunity/\#Tutorial-Reading

${ }^{23}$ Amanna, I. J., Carlson, N. E. \& Slifka, M. K. Duration of humoral immunity to common viral and vaccine antigens. N. Engl. J. Med. 357, 1903-1915 (2007). DOI: 10.1056/NEJMoa066092 https://www.nejm.org/doi/full/10.1056/NEJMoa066092

${ }^{24}$ https://www.cdc.gov/vaccines/covid-19/info-by-product/clinical-considerations.html

${ }^{25}$ https://www.politico.eu/article/who-2nd-dose-of-biontech-pfizer-vaccine-can-be-delayed/

${ }^{26}$ https://timesofmalta.com/articles/view/who-vaccine-advisers-say-second-pfizer-covid-jab-may-be-delayed.843048

${ }^{27}$ https://www.telegraph.co.uk/global-health/science-and-disease/second-dose-pfizer-vaccine-can-delayed-exceptionalcircumstances/ 
